\title{
A valid model for testing and training laparoscopic psychomotor skills
}

\author{
Rudi Campo • Christoph Reising • Yves Van Belle • \\ Joseph Nassif • Peter O'Donovan • \\ Carlos Roger Molinas
}

Received: 3 November 2009 / Accepted: 10 December 2009 / Published online: 2 March 2010

(C) Springer-Verlag 2010

\begin{abstract}
This study aims to evaluate the face and construct validity of the Laparoscopic Skills Testing and Training (LASTT) model, developed by the European Academy of Gynaecological Surgery (EAGS) for assessing laparoscopic psychomotor skills (LPS). This study is designed based on the Canadian Task Force II-1. This study was conducted in workshops organised by the EAGS in 2008 and 2009. One hundred ninety-nine gynaecologists were classified in three groups according to their exposure to laparoscopy (G1: no/little, G2: intermediate, G3: important). Participants performed three repetitions of three exercises (E1: camera navigation, E2: hands-eyes coordi-
\end{abstract}

R. Campo • C. Reising · Y. Van Belle · J. Nassif · P. O’Donovan •

C. R. Molinas

European Academy of Gynaecological Surgery (EAGS),

Leuven, Belgium

R. Campo

Leuven Institute for Fertility and Embryology (LIFE),

Leuven, Belgium

C. Reising

Centre for Surgical Technologies, Katholieke Universiteit Leuven,

Leuven, Belgium

J. Nassif

Institut de Recherche Contre les

Cancers de 1'Appareil Digestif (IRCAD),

Strasbourg, France

P. O’Donovan

Micro Endoscopic Research Innovation

and Training Centre (MERIT),

Bradford, UK

C. R. Molinas $(\bowtie)$

Centre for Gynaecological Endoscopy, Centro Médico La Costa, Asunción, Paraguay

e-mail: roger.molinas@lacosta.com.py nation, E3: bimanual coordination) with measurable objectives to accomplish within a limited time frame. The face validity of the model was assessed by an 11-item questionnaire using a $10-\mathrm{cm}$ visual analogue scale. Q1Q8 evaluated its capacity for testing and training LPS and Q9-Q11 its relevance for actual laparoscopic surgery. The score of each exercise was obtained by dividing the time to correct performed exercise by the number of objectives effectively accomplished. The correlation between E1-E3 scores and the level of exposure to laparoscopy was evaluated, and the scores of the different groups were compared to assess the construct validity. Participants gave a favourable opinion about the model without inter-group differences. In E1-E3, the scores correlated with the level of previous exposure to laparoscopic procedures, moreexperienced participants achieving better results than lessexperienced participants. The data demonstrate the face and construct validity of the LASTT model, suggesting that it can be a useful tool for training and evaluation of LPS in surgical disciplines that perform laparoscopic procedures.

Keywords Laparoscopic psychomotor skills · Testing model $\cdot$ Training model $\cdot$ Face validity $\cdot$ Construct validity

\section{Introduction}

The education methods for training in gynaecological surgery are being challenged by different forces and influences, such as the boundaries of the traditional apprentice-tutor model, the ethical objective to limit patient morbidity and error rate during surgery and the continuous pressure on cost effectiveness. Against such challenges, it is mandatory that objective measurable levels of practical skills should be established and validated prior to gynaecological surgery. 
Some aspects of this complex problem of training, education and certification in laparoscopic surgery were recently addressed in a very striking way by the Dutch Ministry of Health (http://www.igz.nl/actueel/nieuwsberichten/mic) because the health inspection found an unacceptable amount of serious complications in common laparoscopic procedures. The inspection assessed the manner in which patient safety is assured and the quality of the procedures in terms of practitioner skills and training. The report concluded that training in laparoscopic techniques was inadequately structured and that it is a matter of concern that the standards which a future laparoscopist must meet in order to operate, either independently or under supervision, have not been adequately established.

It seems obvious, but not yet implemented, that a future laparoscopist should possess objective measurable theoretical and practical skills to be able to enter in a one-to-one teaching process in the operating room (OR). Different models have been proposed for this aim. In vitro models (e.g. trainer box, virtual reality) allow the monitoring of skills acquisition in a relaxed and controlled environment [1-3]. Trainer boxes are relatively cheap and accessible [4] and are as effective as virtual reality models [5], but unfortunately scientific validation of most of them remains insufficiently studied and underreported.

A new in vitro model, called the Laparoscopic Skills Testing and Training (LASTT) model, aimed to train and measure essential laparoscopic psychomotor skills (LPS; i.e. camera navigation, hands-eyes coordination, bimanual coordination), has recently been developed by the European Academy of Gynaecological Surgery (EAGS) [6]. It has been suggested that this model can be a cost-effective tool for continuous training and evaluation of LPS in all surgical disciplines that perform laparoscopic procedures because it is tutor independent, relatively cheap and suitable for any trainer box. Its feasibility and construct validity (i.e. its capacity to distinguish between experienced and inexperienced surgeons) have been well demonstrated [6].

This study was designed to evaluate the face validity (the realism of the method) and to confirm the construct validity of the LASTT model in a large population of residents and specialists in OB\&GYN attending test sessions organised by the EAGS. The participants were classified according to their level of exposure to laparoscopic procedures, aiming to evaluate the correlation between the clinical experience and the proficiency in the essential LPS.

\section{Methods}

Participants and venue

The study enrolled residents and gynaecologists with different levels of experience in laparoscopic surgery $(n=$
199) who voluntarily attended workshops organised by the EAGS during the 20th European Congress on Obstetrics and Gynaecology (EBCOG) in Lisbon, Portugal, in 2008 $(n=56)$, the 24th Annual Meeting of the European Society of Human Reproduction and Embryology (ESHRE) in Barcelona, Spain, in $2008(n=58)$, the Workshop on Laparoscopic Hysterectomy in Tübingen, Germany, in $2008(n=24)$, the Laparoscopic Suturing Course in Leuven, Belgium, in $2009(n=7)$, the 19th European Meeting of the European Network for Trainees in Obstetrics and Gynaecology (ENTOG) in Budapest, Hungry, in $2009(n=12)$ and the 30th Congress of the Spanish Society of Gynaecology and Obstetrics (SEGO) in Barcelona, Spain, in $2009(n=42)$.

Instruments and materials

The LASTT model, suitable for performing three standardised exercises, as described elsewhere [6], was used. The insert, with the relevant materials for the different exercises, was inserted into the Szabo trainer box (Karl Storz, Tuttlingen, Germany). The exercises were performed with standard instruments $\left(10 \mathrm{~mm} 0^{\circ} / 30^{\circ}\right.$ optic, 5 -mm Kelly forceps and Matkowitz forceps) and an all-in-one (monitor, light source and video camera) laparoscopic tower (Telepack, Karl Storz, Tuttlingen, Germany).

\section{Laparoscopic exercises (E)}

E1-camera navigation This exercise was used to evaluate the participant's ability to navigate a laparoscopic camera with a $30^{\circ}$ optic. This was done by measuring their ability to identify 14 different targets placed at different sites in the LASTT model [6]. Each target included a large symbol identifiable from a panoramic viewpoint and a small symbol only identifiable from a close-up viewpoint (Fig. 1a). The exercise started by identifying the large symbol on the first target (i.e. 1) and then the small symbol situated next to it, which had to be shown on the centre of the screen. This small symbol indicated the next large symbol to be identified. Following this order, the participant continued until the identification of the small symbol on the last target (i.e. end).

E2-hands-eyes coordination This exercise was used to evaluate the participant's ability to navigate a laparoscopic camera with a $0^{\circ}$ optic with the non-dominant hand (NDH) and to handle a laparoscopic forceps with the dominant hand (DH). This was done by measuring their ability to grasp and transport six pre-defined objects to six predefined targets in the LASTT model, which was fitted with coloured objects $(5 \times 4-\mathrm{mm}$ open cylinders $)$ and coloured targets $(10 \times-1 \mathrm{~mm}$ nails) [6]. The matched targets and objects were identifiable by colour (Fig. 1b). The exercise 
Fig. 1 The LASTT model. a Setup for E1 (camera navigation). b Setup for E2 (hands-eyes coordination). c Setup for E3 (bimanual coordination)
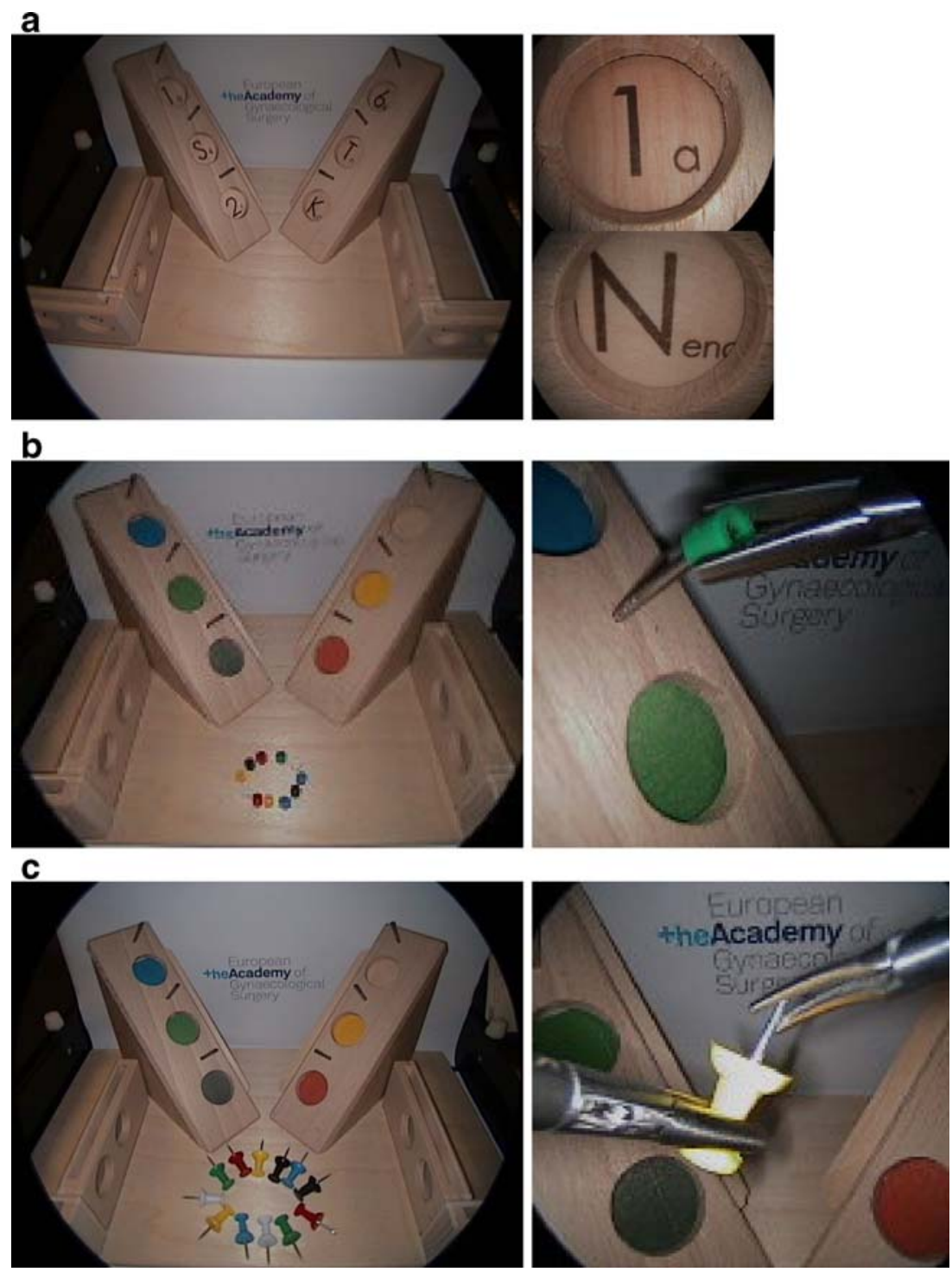

started by identifying a target and an object of the same colour. Then, the object was grasped, transported and introduced onto the relevant nail. Only when the participant succeeded in introducing the open cylinder onto the matched nail was he/she allowed to continue with the next object of a different colour.

E3-bimanual coordination This exercise was used to evaluate the participant's ability to handle laparoscopic forceps simultaneously with the DH and the NDH. This was done by measuring the participant's ability to grasp six pre-defined objects with the $\mathrm{DH}$ and re-grasp and transport them with the NDH to six pre-defined targets on the LASTT model, which was fitted with coloured objects $(10 \times 5-\mathrm{mm}$ push pins with a tail of $10 \mathrm{~mm})$ and coloured targets (20-mm holes) [6]. The matched targets and objects were identifiable by colour (Fig. 1c). The exercise started by identifying a target and an object of the same colour.
Then, the push pin was grasped by the head with the Matkowitz forceps (DH) and exposed to the Kelly forceps (NDH), with which it was re-grasped by the tail, transported and introduced into its target. Only when the participant succeeded with the introduction of an object into its target was he/she allowed to continue with the others.

\section{Experimental design, scoring and statistics}

Standardised sessions with simultaneous working stations were organised during the above-mentioned meetings. Each station had a tutor and two participants at a time. At the beginning of the session, participants completed a survey about demographic (Table 1) and previous exposure to gynaecological laparoscopy using the classification of the European Society for Gynaecological Endoscopy. This classification establishes four levels of procedures: first 
Table 1 Participants' demographics

\begin{tabular}{|c|c|c|c|}
\hline & \multicolumn{3}{|l|}{ Group } \\
\hline & G1 $(n=85)$ & $\mathrm{G} 2(n=44)$ & G3 $(n=63)$ \\
\hline Age (mean $\pm \mathrm{SD})$ & $30 \pm 7$ & $39 \pm 10$ & $42 \pm 9$ \\
\hline \multicolumn{4}{|l|}{ Gender } \\
\hline Males & $22(26 \%)$ & $18(41 \%)$ & $40(63 \%)$ \\
\hline Females & $63(74 \%)$ & $26(59 \%)$ & $23(37 \%)$ \\
\hline \multicolumn{4}{|l|}{ Training status } \\
\hline Residents in OB\&GYN & $41(80 \%)$ & $19(43 \%)$ & $14(22 \%)$ \\
\hline Specialists in OB\&GYN & $17(20 \%)$ & $25(57 \%)$ & $49(78 \%)$ \\
\hline \multicolumn{4}{|l|}{ Dominant hand side } \\
\hline Right & $81(95 \%)$ & $41(91 \%)$ & $58(92 \%)$ \\
\hline Left & $4(5 \%)$ & $3(9 \%)$ & $5(8 \%)$ \\
\hline
\end{tabular}

level (basic), second level (intermediate), third level (advanced) and fourth level (special procedures) [6]. For each level, the numbers of procedures performed were recorded and then scored in the following categories: no procedures (score 0), 1-30 procedures (score 1), 31-50 procedures (score 2 ) and more than 50 procedures (score 3 ). The scores obtained in each level were added, giving a final score ranging from 0 to 12 . This score, resulting from many possible combinations, represents the amount of laparoscopic procedures to which an individual was exposed to.

At the time of data analysis, participants were classified in three groups $(\mathrm{G})$. G1 comprises those with no or very little exposure to laparoscopy (final score 0 or 1). G2 comprises those with limited exposure to laparoscopy (final score 2 or 3 ). G3 comprises those with important exposure to laparoscopy (final score equal to or more than 4).
The exercises were performed in chronological order: E1, E2 and finally E3. Full explanation and demonstration were given at the beginning of each exercise. For each exercise, participants performed three repetitions in alternate order with his/her partner. This number was determined to avoid the major learning effect observed after three repetitions and to be consistent with previous studies [6]. For each repetition, the time was limited to $120 \mathrm{~s}$ for E1 and to $180 \mathrm{~s}$ for E2 and E3. This limit was based on previous observations that a large amount of people could finalise the task within this period and on the obvious time restrictions encountered during large medical meeting.

In each repetition, the numbers of objectives actually achieved were recorded (i.e. targets identified for E1 and objects transported for E2 and E3). When any objectives were accomplished, a value of 0.5 was assigned. The measurement of the exercises was based on the time to correct performed exercise (TCPE), which reflects errors and economy of movement in the result and as such engages an accuracy assurance. Since some participants may not finalise the task in the assigned time, the final score was obtained by dividing the actual time used by the number of objectives effectively accomplished. The average values of the triplicate observations were used for statistical analysis. The scores were not normally distributed, and therefore median values (inter-quartile range) are presented. The correlation between the self-reported level of exposure to laparoscopy and the LPS was evaluated with the Spearman test. Intergroup differences were evaluated by the Kruskal Wallis test (with Dunn's correction for multiple-comparison tests).

To assess the face validity of the LASTT model, participants were asked to respond to a questionnaire with 11 questions (Q; Table 2) using a 10-cm visual analogue

Table 2 Questionnaire to test the face validity of the model and the exercise

\begin{tabular}{|c|c|c|}
\hline Questions & Score of the total population $(n=192)$ & \\
\hline \multicolumn{3}{|c|}{ Face validity of the model for testing and training LPS } \\
\hline Q1 & Ability to test camera navigation (E1) & $8.4(7.6-9.1)$ \\
\hline Q2 & Ability to train camera navigation (E1) & $9.0(8.0-9.8)$ \\
\hline Q3 & Ability to test hands-eyes coordination (laparoscopic forceps handling with the DH) (E2) & $8.6(7.6-9.6)$ \\
\hline Q4 & Ability to train hands-eyes coordination (laparoscopic forceps handling with the DH) (E2) & $8.4(7.0-9.5)$ \\
\hline Q5 & Ability to test bimanual coordination (laparoscopic forceps handling with the DH and NDH simultaneously) (E3) & $8.6(7.7-9.7)$ \\
\hline Q6 & Ability to train bimanual coordination (laparoscopic forceps handling with the DH and NDH simultaneously) (E3) & $9.0(7.9-9.8)$ \\
\hline Q7 & Overall value of the model as a testing tool & $8.8(8.0-9.4)$ \\
\hline Q8 & Overall value of the model as a training tool & $9.0(8.0-9.8)$ \\
\hline \multicolumn{3}{|c|}{ Face validity of the model for actual laparoscopic surgery } \\
\hline Q9 & Relevance of the model for actual laparoscopic surgery & $8.4(7.2-9.3)$ \\
\hline Q10 & Realism of the model to simulate the female pelvis & $7.3(5.3-9.0)$ \\
\hline Q11 & Realism of the model to simulate the movements required to perform laparoscopic surgery in the pelvis & $8.4(7.0-9.5)$ \\
\hline
\end{tabular}

Median (inter-quartile range) scores of a 10-cm VAS (0: not realistic/good/useful; 10: very realistic/good/useful) are presented 
scale (VAS; 0: not realistic/good/useful; 10: very realistic/ good/useful). Q1-Q8 examined the usefulness of the different exercises and of the overall model in terms of their testing and training capacities for LPS. These questions were answered at the end of each exercise (Q1Q6) and at the end of the sessions (Q7-Q8). Q9-Q11 examined the usefulness of the model in terms of its realism and relevance to laparoscopic surgery and were answered at the end of the session. The scores were not normally distributed, and therefore median values (inter-quartile range) are presented. Inter-group differences were evaluated by the Kruskal Wallis test (with Dunn's correction for multiple-comparison tests).

All statistical comparisons were performed with the GraphPad Prism Software and two- tailed $P$ values $<0.05$ were considered significant.

\section{Results}

Seven participants did not perform all the assigned tests and were therefore excluded from the study. The remaining 192 participants were classified according to their level of exposure to laparoscopic surgery: G1 $(n=85), \mathrm{G} 2(n=44)$ and G3 $(n=63)$. Their demographics are reported in Table 1.

\section{Face validity}

The response rate to the questionnaire was of $97.7 \%$ (97.3\% in G1, $97.5 \%$ in G2 and $98.3 \%$ in G3). The total population (Table 2), as well as the three groups separately (Fig. 2), gave a favourable response to this model.
The first six questions (Q1-Q6), in which the individual exercises were evaluated in terms of their testing and training capacities, were well validated by all participants, all receiving a score above 8 , without inter-group differences. This evaluation of the individual exercises was reflected in the overall assessment of the model. Indeed, all participants considered that the model was good for testing purposes (Q7), with a score of $8.8(8.0-9.4)$, and for training purposes (Q8), with a score of $9.0(8.0-9.8)$, without inter-group differences.

In the last three questions, the relevance of the model for actual laparoscopic surgery (Q9) and its realism to simulate the female pelvis (Q10) and to simulate the movements required to perform laparoscopic surgery in the pelvis (Q11) were evaluated. Q9 and Q11 were positively judged, with a score of $8.4(7.2-9.3)$ and 8.4 (7.0-9.5), respectively. Q10, however, received a score of 7.3 (5.3-9.0) only, being the questions which received the lowest validation. In the three questions, no inter-group differences were detected.

\section{Construct validity}

In all exercises, participants previously exposed to a larger amount of laparoscopic procedures achieved better results than participants exposed to fewer procedures.

For camera navigation (E1, Fig. 3), a negative correlation $(r=-0.39 ; P<0.0001)$ between the scores and the level of exposure to laparoscopy was found. The scores were 14 (9-21) for G1, $11(8-18)$ for $\mathrm{G} 2$ and $8(7-10)$ for G3. G3 scored better than $\mathrm{G} 1(P<0.001)$ and $\mathrm{G} 2(P<0.01)$. The differences between $\mathrm{G} 2$ and G1 were not significant.

For hands-eyes coordination (E2, Fig. 4), a negative correlation $(r=-0.42 ; P<0.0001)$ between the scores and the level of exposure to laparoscopy was found. The scores
Fig. 2 Median (inter-quartile range) scores of a questionnaire assessing the face validity of the LASTT model using a $10-\mathrm{cm}$ VAS ( 0 : not realistic/good/ useful; 10: very realistic/good/ useful). Participants were grouped according to their level of exposure to laparoscopic surgery in three groups: G1 (no or little exposure, green bars), G2 (intermediate exposure, yellow bars) and G3 (important exposure, orange bars)

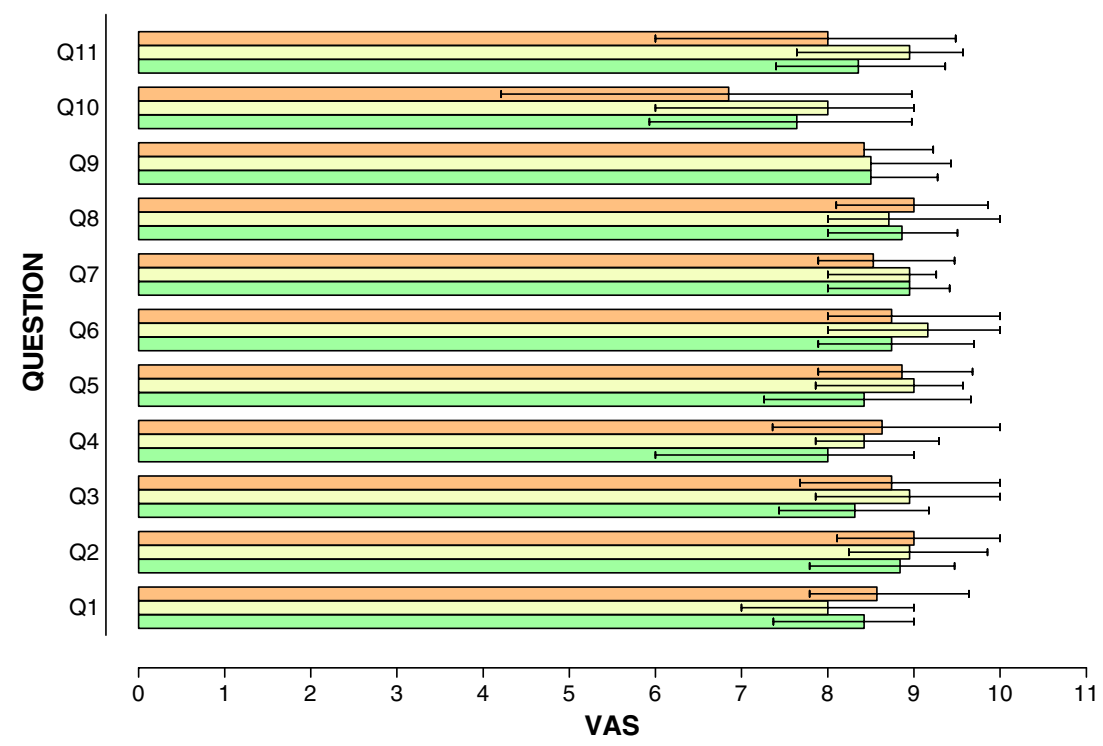




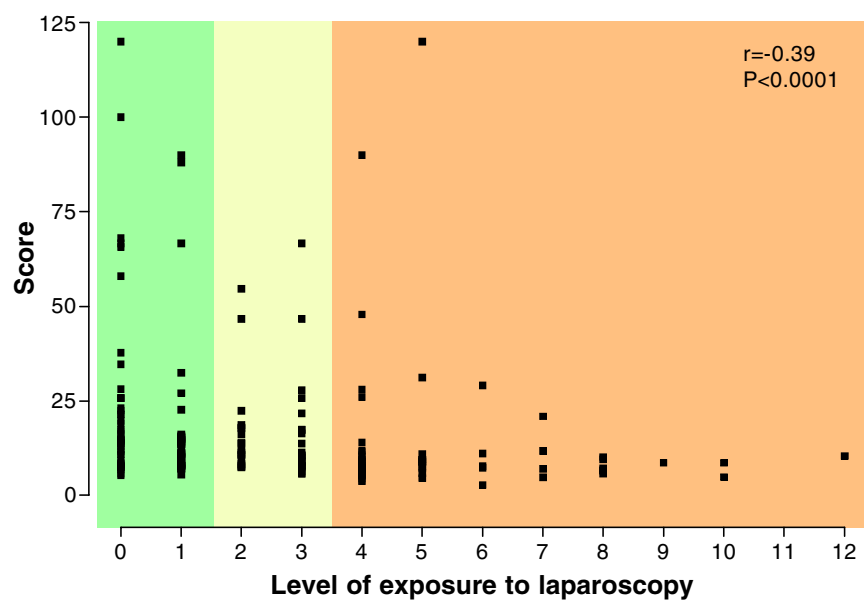

Fig. 3 Exercise 1 (camera navigation). The ability to identify 14 different targets placed at different sites in the LASTT model was evaluated. The left graph shows the scores of the participants in function to their exposure to laparoscopic surgery (ranged from 0 to

were 45 (31-91) for G1, 33 (21-48) for G2 and 29 (20-40) for G3. G3 scored better than G1 $(P<0.001)$ but not $\mathrm{G} 2(P=$ NS). G2 scored better than G1 $(P<0.01)$.

For bimanual coordination (E3, Fig. 5), a negative correlation $(r=-0.44 ; P<0.0001)$ between the scores and the level of exposure to laparoscopy was found. The scores were 40 (30-64) for G1, 31 (26-41) for G2 and 25 (16-34) for G3. G3 scored better than G1 $(P<0.001)$ and G2 $(P<$ $0.01)$. G2 scored better than G1 $(P<0.05)$.

\section{Discussion}

In the classic apprenticeship system of "see one, do one, teach one", feedback is directly provided during surgery in

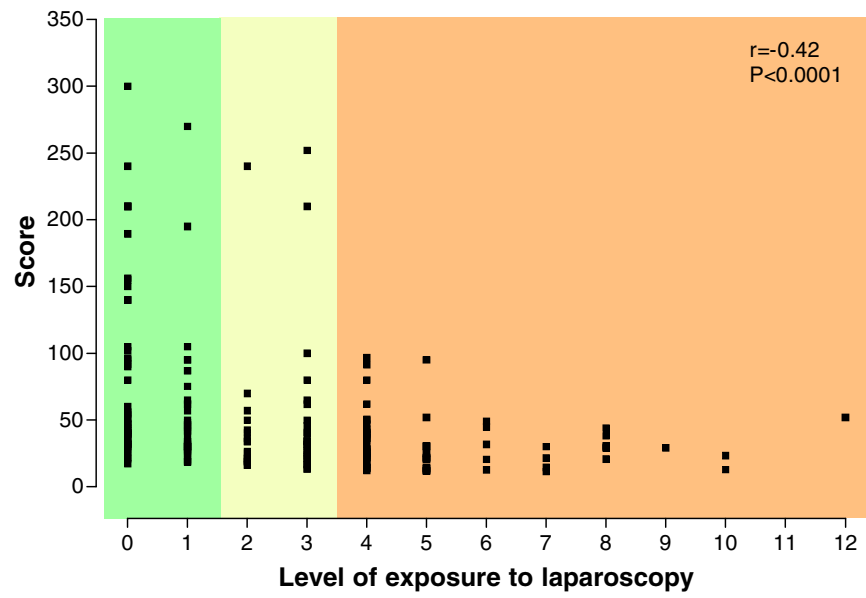

Fig. 4 Exercise 2 (hands-eyes coordination). The ability to grasp and transport six pre-defined objects to six pre-defined targets with the DH in the LASTT model was evaluated. The left graph shows the scores of the participants in function to their exposure to laparoscopic surgery (ranged

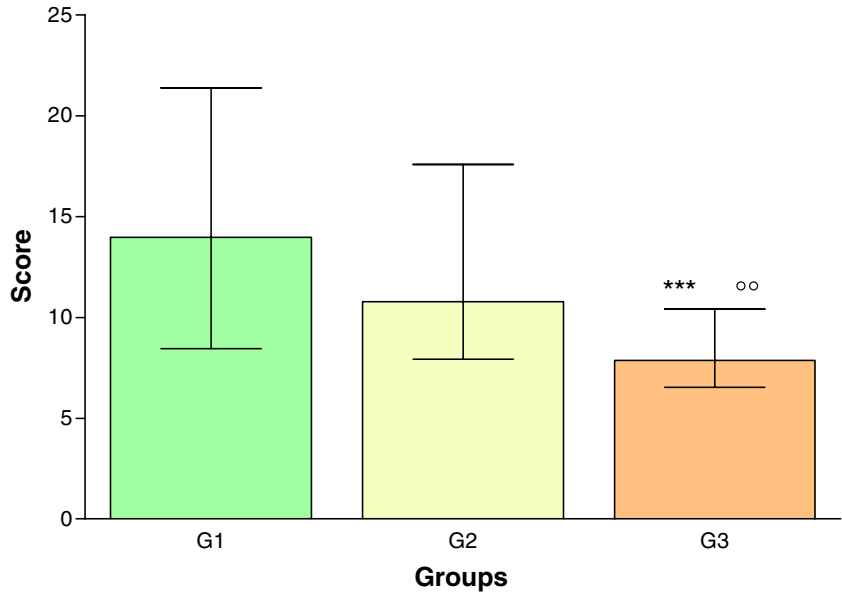

12). The right graph shows the median (inter-quartile range) scores of the three groups (G1: no or little exposure to laparoscopy, G2: intermediate exposure to laparoscopy, G3: important exposure to laparoscopy). ${ }^{* * *} P<0.001$ (G1 vs. G3); ${ }^{\circ} P<0.01$ (G2 vs. G3)

the OR, and surgery is learnt by the student through simple observation and later imitation of the actions of a skilled mentor. However, there are critical factors for the current use of this model, such as the necessity of a high volume of surgical procedures, the availability of a sufficient number of skilled mentors, the limitation of resident working hours and some ethical and financial constraints.

Training in laparoscopic surgery increases existing problems because besides the typical surgical skills the trainee has to acquire the LPS (i.e. hand-eye coordination, camera navigation, remote handling of instruments without tactile feedback and fine motor skills to deal with the fulcrum effect and the lever forces of the long instrument). The acquisition of these skills through the classic apprenticeship system seems not only impossible but also ethically

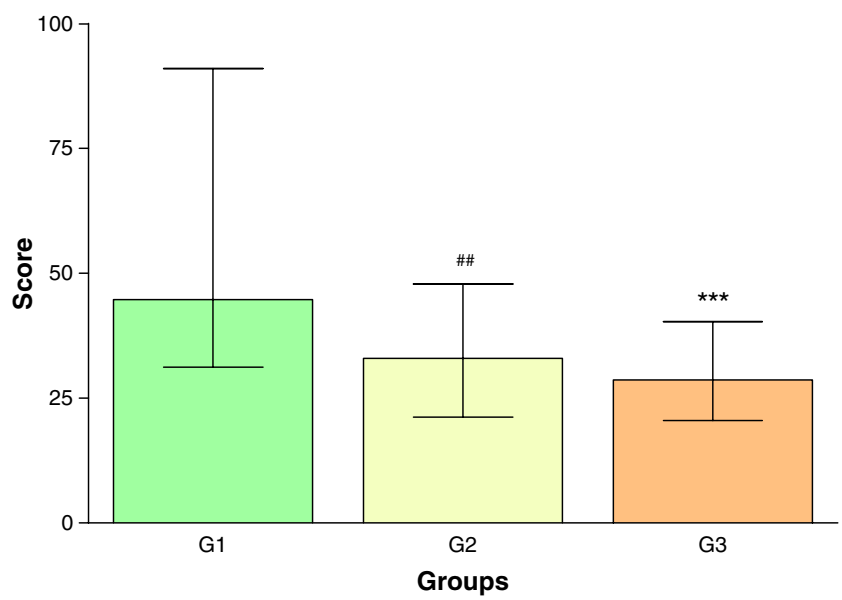

from 0 to 12). The right graph shows the median (inter-quartile range) scores of the three groups (G1: no or little exposure to laparoscopy, G2: intermediate exposure to laparoscopy, G3: important exposure to laparoscopy). ${ }^{\# \#} P<0.01$ (G1 vs. G2); ${ }^{* * *} P<0.001$ (G1 vs. G3) 


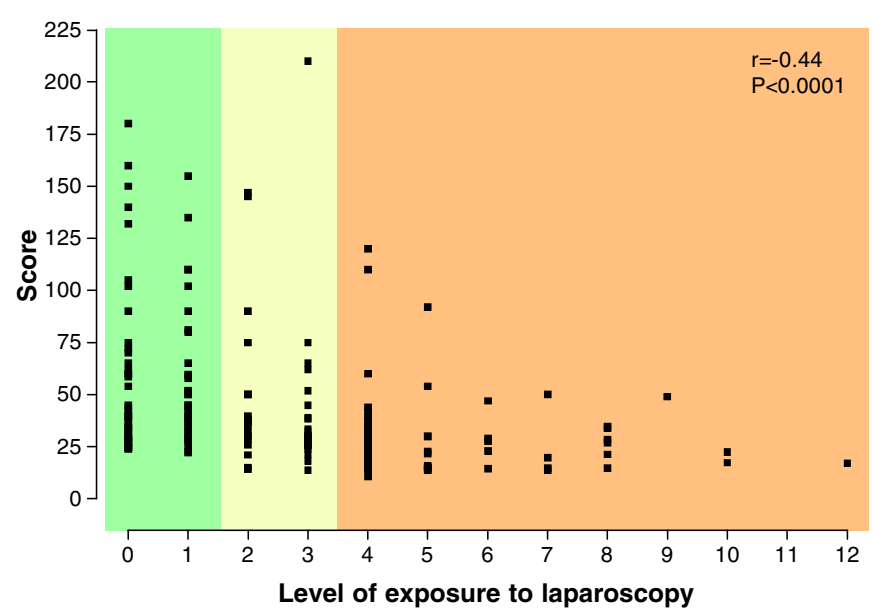

Fig. 5 Exercise 3 (bimanual coordination). The ability to grasp six pre-defined objects with the $\mathrm{DH}$ and re-grasp and transport them with the NDH to pre-defined targets in the LASTT model was evaluated. The left graph shows the scores of the participants in function to their exposure to laparoscopic surgery (ranged from 0 to 12). The right

unacceptable, as it might increase the complication rate of laparoscopic procedures. To shorten learning curves and to reduce accidents and complications, specific LPS and some typical surgical skills, such as suturing or knot tying, must be learnt outside the OR.

Although several training devices and methods for laparoscopic skills acquisition have been reported [7-14], most studies focused on models that recreate operative conditions and very few on the specific LPS. Virtual reality models have been proposed in this regard but, as they are still very expensive, a simple and broad implementation (not only at specialised centres) is not feasible today. The EAGS has developed the LASTT model, which focuses on the acquisition and measurement of three essential LPS (i.e. camera navigation, hand-eye coordination and bimanual coordination) [6]. The experience gathered so far demonstrates that this model can be used as an insert in a conventional trainer box and is feasible for large-scale adaptation. Furthermore, its capacity to distinguish between novices and experts (i.e. construct validity) has been recently demonstrated [6].

The present study extends these observations by demonstrating the face validity of the LASTT model, which indicates the appropriateness of the method on "its face value" (the resemblance of a test task to the actual clinical task). Since the LASTT model does not simulate nor represent any specific laparoscopic procedure (there is no specific clinical task involved), the assessment of its face validity should be very cautious. The model aims to train and measure LPS only, which is a concept not easily understood by physicians without experience in laparoscopic surgery who want to rapidly acquire proficiency to perform specific laparoscopic procedures and not only to

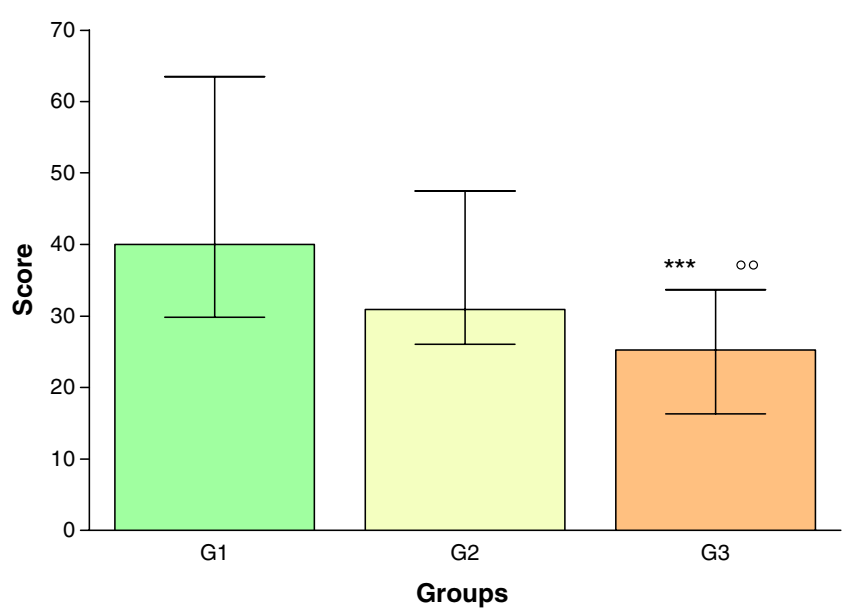

graph shows the median (inter-quartile range) scores of the three groups ( $G 1$ : no or little exposure to laparoscopy, G2: intermediate exposure to laparoscopy, G3: important exposure to laparoscopy). $* * * P<0.001$ (G1 vs. G3); ${ }^{\circ} P<0.01$ (G2 vs. G3)

navigate a camera or to handle an instrument. The total population, as well as the three study groups separately, gave a favourable opinion about the face validity of the model for testing and training LPS and also about its face validity for actual laparoscopic surgery. The realism of the model to simulate the female pelvis was, however, the least validated, which is not surprising taking into account that the LASTT model does not pretend to resemble the anatomy of a female pelvis but only the spatial distribution and orientation of its different planes and angles.

To assess the face validity of the study, it is important to be aware that the opinions of both types of participants might be influenced by several factors [15]. To what extent the less-experienced laparoscopists are just polite or feel obliged to fill in a questionnaire in exchange for a chance to "play" with the model is difficult to measure. Another effect that must be considered is the "Pygmalion effect" named after Pygmalion, a king figure from ancient Greek mythology, who carved a sculpture out of stone so skillfully that he fell in love with it. In this setting, it might be that the answers of the experienced, but especially of the lessexperienced laparoscopists, were influenced by the mere enthusiasm of the LASTT developers, who took part in the workshops and gave the demonstration. The lessexperienced laparoscopists may be particularly susceptible to give favourable responses because they have been around for less time in the working field of surgery and because they are relatively unprotected against the tempting display of the model and instruments by industry. On the other hand, the more-experienced laparoscopists, trained by the classical apprentice-tutor model, may be more conservative in their opinion of surgical training novelties. Nevertheless, even if these phenomena are of influence, 
they were not reflected in the outcomes of the study as manifested in the strong uniformity of opinion amongst groups.

In addition to demonstrating the face validity of the LASTT model, this study confirms its construct validity in another population and demonstrates the construct validity of an adapted scoring system. In the previous studies, each exercise was evaluated with different scoring systems in which the time and/or the number of objectives to accomplish were limited [6]. This resulted in data with very large variability and in a poorly reproducible scoring system. This scoring was modified to be more easily and universally applied. In this study, the maximum time allowed for each repetition was limited for practical organisational reasons, and quantifiable objectives were defined for each task. The main parameter measured was TCPE (i.e. the time required to finalise successfully the task). It was likely, however, that some participants would not be able to finalise the exercise within the time frame. To be able to include the data of those participants, the TCPE score was adapted, and the final score was obtained by dividing the time used by the number of objectives effectively accomplished.

It must be admitted that other factors that could influence the performance of the tasks, such as errors and economy of the movements, were not directly measured. It was assumed that with some limitations they will affect anyway in the TCPE score and thus be reflected in the final score. In spite of these limitations, this scoring system is similar for the three tasks and allows for the comparison of scores obtained at different locations, in which the time dedicated to the task could be different. Furthermore, it has the advantage of being objective, tutor independent and useful for self-assessment.

Besides the face and construct validity of the LASTT model, this study also demonstrates a strong correlation between the self-reported clinical exposure to laparoscopic surgery and the three LPS evaluated. Indeed, the very poor LPS (with large inter-individual variability) observed in most participants with little exposure to laparoscopy become progressively better (with small inter-individual variability) in function to the amount of laparoscopic procedures performed. Although the study did not pretend to detect the proficiency level of the LPS (participants performed three repetitions only), the data indicate that if novices want to achieve the experts' LPS levels by clinical exposure in the OR only an enormous amount of laparoscopic procedures would be necessary. This would be not only unethical but also virtually impossible in the current residency programmes and confirms the necessity of validated pre-graduate (outside the OR) training programmes.

The data obtained in this and in the previous study [6] suggest that the LASTT model can be a useful tool for training and assessing the LPS of the residents in their own hospitals/universities. It provides tutors an objective method for evaluation of basic laparoscopic skills, which together with the theoretical knowledge of anatomy, laparoscopic principles and instrumentation and OR functioning, are the essential pre-requisites before an in vivo OR training programme with the apprentice tutor model can be started.

Furthermore, the LASTT model can be used as research tool for evaluating the different parameters of the learning curve (e.g. length, shape, slope, plateau, etc.) in order to optimise the acquisition and retention of the laparoscopic skills in a specific group and/or an individual trainee and more importantly for the establishment of performance standards [16-18].

Acknowledgements The study was possible thanks to a scientific grant of the European Academy of Gynaecological Surgery. We acknowledge the staff of the EAGS, especially M. De Rocker, K. Goeminne and K. Carmeliet, for their organisational help.

We would like to thank the organisers of the different congresses who provided us with a platform for performing large-scale evaluation: W. Holzgreve, Z. Novak and J. Martinez de Oliveira (EBCOG, Lisbon 2008), S. Gordts and M. Gergolet (ESHRE, Barcelona 2008), D. Wallwiener and E. Solomayer (Universitäts Frauenklinik Tübingen 2008), A. Wattiez and Y. Van Belle (The House of the European Academy, Leuven 2009), E. Werner and T. Orosz (ENTOG, Budapest 2009) and F. D. Rodriguez Morante and Carmona Herrera (SEGO, Barcelona 2009).

We thank Karl Storz Company (Tuttlingen, Germany) for their support to the academy and for providing the necessary materials at the different locations.

\section{References}

1. Larsen CR, Grantcharov T, Aggarwal R, Tully A, Sorensen JL, Dalsgaard T, Ottesen B (2006) Objective assessment of gynecologic laparoscopic skills using the LapSimGyn virtual reality simulator. Surg Endosc 20:1460-1466

2. Undre S, Darzi A (2007) Laparoscopy simulators. J Endourol 21:274-279

3. McDougall EM (2007) Validation of surgical simulators. J Endourol 21:244-247

4. Katz R (2006) Methods of training using pelvic trainers. Curr Urol Rep 7:100-106

5. Munz Y, Kumar BD, Moorthy K, Bann S, Darzi A (2004) Laparoscopic virtual reality and box trainers: is one superior to the other? Surg Endosc 18:485-494

6. Molinas CR, De Win G, Ritter O, Keckstein J, Miserez M, Campo R (2008) Feasibility and construct validity of a novel laparoscopic skills testing and training model. Gynecol Surg 5:281-290

7. Aggarwal R, Boza C, Hance J, Leong J, Lacy A, Darzi A (2007) Skills acquisition for laparoscopic gastric bypass in the training laboratory: an innovative approach. Obes Surg 17:19-27

8. Molinas CR, Binda MM, Mailova K, Koninckx PR (2004) The rabbit nephrectomy model for training in laparoscopic surgery. Hum Reprod 19:185-190

9. Schijven MP, Jakimowicz JJ (2003) Introducing the Xitact LS500 laparoscopy simulator: toward a revolution in surgical education. Surg Technol Int 11:32-36 
10. Ayodeji ID, Schijven M, Jakimowicz J, Greve JW (2007) Face validation of the Simbionix LAP Mentor virtual reality training module and its applicability in the surgical curriculum. Surg Endosc 21:1641-1649

11. Maithel S, Sierra R, Korndorffer J, Neumann P, Dawson S, Callery M, Jones D, Scott D (2006) Construct and face validity of MIST-VR, Endotower, and CELTS: are we ready for skills assessment using simulators? Surg Endosc 20:104-112

12. Korndorffer JR Jr, Stefanidis D, Scott DJ (2006) Laparoscopic skills laboratories: current assessment and a call for resident training standards. Am J Surg 191:17-22

13. Fried GM (2008) FLS assessment of competency using simulated laparoscopic tasks. J Gastrointest Surg 12:210-212

14. Vassiliou MC, Ghitulescu GA, Feldman LS, Stanbridge D, Leffondre K, Sigman HH, Fried GM (2006) The MISTELS program to measure technical skill in laparoscopic surgery: evidence for reliability. Surg Endosc 20:744-747

15. Schijven M, Jakimowicz J (2002) Face-, expert, and referent validity of the Xitact LS500 laparoscopy simulator. Surg Endosc $16: 1764-1770$

16. Kolkman W, van de Put MAJ, Wolterbeek R, Trimbos JBMZ, Jansen FW (2008) Laparoscopic skills simulator: construct validity and establishment of performance standards for residency training. Gynecol Surg 5:109-114

17. Huirne JAF, Kennedy R, Stones RE, Brolmann HAM (2008) What is the impact of surgical expertise and how to get it? Gynecol Surg 5:265-267

18. Jansen FW, Kolkman W (2008) Implementation difficulties of advanced techniques in gynecological laparoscopy. Gynecol Surg $5: 261-264$ 